\title{
Extent of Carotid Sinus Regulation of the Myocardial Contractile State in Conscious Dogs
}

\author{
Stephen F. Vatner, Charles B. Higgins, Dean Frankinn, and \\ EUgene Braunwald \\ From the Department of Medicine, University of California, San Diego, \\ School of Medicine, and Scripps Clinic and Research Foundation, \\ La Jolla, California 92037
}

A B S T R A C T The effects of bilateral carotid artery occlusion (BCO) and carotid sinus nerve stimulation (CSNS) on left ventricular (LV) pressure (P), diameter (D), velocity of contraction (V), rate of change of pressure $(\mathrm{dP} / \mathrm{dt})$, and cardiac output were studied in conscious dogs instrumented with ultrasonic diameter gauges, miniature pressure gauges, and aortic electromagnetic flow transducers. The effects of $\mathrm{BCO}$ and CSNS were also studied after autonomic blockade and were compared to similar alterations in pressure produced by norepinephrine, methoxamine, and nitroglycerin. When heart rate was maintained constant with atrial stimulation, BCO had little effect on ventricular contractility, increasing isolength systolic pressure (LV $\mathrm{P}_{1 \mathrm{so}}$ ) by $36 \%$ while isolength velocity of myocardial shortening $\left(\mathrm{V}_{1 \mathrm{so}}\right)$ decreased by $12 \%$ and $(\mathrm{dP} / \mathrm{dt}) / \mathrm{P}$ fell by $8 \%$. These effects could be explained largely by vasoconstriction, since elevating systolic pressure with methoxamine produced similar results, while norepinephrine increased $\mathrm{V}_{1 \text { so }}$ by $36 \%$ and $(\mathrm{dP} / \mathrm{dt}) / \mathrm{P}$ by $56 \%$. CSNS produced directionally opposite results from $\mathrm{BCO}$; it decreased $\mathrm{P}_{1 \text { so }}$ by $15 \%, \mathrm{~V}_{\text {iso }}$ increased by $11 \%$, while $(\mathrm{dP} / \mathrm{dt}) / \mathrm{P}$ remained almost constant. These effects may be explained largely by vasodilatation since reducing systolic pressure to the same level with nitroglycerin produced similar results. When peripheral vasoconstriction was minimized by phenoxybenzamine pretreatment, $\mathrm{BCO}$ produced a slight positive inotropic effect ( $\mathrm{P}_{1 \mathrm{so}}$ increased by $8 \%, \mathrm{~V}_{\mathrm{iso}}$ by $4 \%$, and (dp/

This study was presented in part at the 44th Annual Scientific Sessions of the American Heart Association, Anaheim, Calif., November 1971. An abstract appeared in a Supplement to Circulation, October 1971.

Dr. Higgins is a Surgical Scholar supported by U. S. Public Health Service Grant GM 01559.

Received for publication 18 August 1971 and in revised form 12 November 1971.
dt)/P by $10 \%$ ), while CSNS produced a slight negative inotropic effect $\left(\mathrm{P}_{\mathbf{1}}\right.$ o decreased by $3 \%$, V 1 so decreased by $5 \%$, and $(\mathrm{dP} / \mathrm{dt}) / \mathrm{P}$ by $7 \%)$.

Thus, in the normal, healthy, conscious dog, the carotid sinuses exert relatively little control of the inotropic state of the left ventricle; moreover, this small inotropic action is masked by the more powerful effects on peripheral resistance.

\section{INTRODUCTION}

The baroreceptors, and the carotid sinus reflex in particular, are thought to exert considerable control over the myocardial contractile state (1-8); an increase in carotid sinus pressure is thought to reduce contractility reflexly, while carotid sinus hypotension produces the opposite effect. Although an important role for carotid sinus control of contractility has been found by most investigators (1-8), some studies have suggested that this may not be the case $(9,10)$. Previous investigations of this important subject have been conducted in anesthetized animals with open chests (1-10). However, there is little information regarding the carotid sinus control of the myocardial contractile state in the intact conscious animal, in which all circulatory control mechanisms are intact. We have previously observed that general anesthesia radically alters the coronary, peripheral vascular, and chronotrophic responses to carotid sinus nerve stimulation (CSNS) ${ }^{1}$ (11), and have also demonstrated that it substantially depresses the myocardial contractile state, resulting in an augmented response to

\footnotetext{
' Abbreviations used in this paper: $\mathrm{BCO}$, bilateral carotid artery occlusion; CSNS, carotid sinus nerve stimulation; $\mathrm{D}$, diameter; $\mathrm{dD} / \mathrm{dt}$, rate of change of diameter; $\mathrm{dP} / \mathrm{dt}$, rate of change of pressure; $L V$, left ventricular; $\mathrm{P}_{1 \mathrm{so}}$, isolength systolic pressure; $\mathrm{V}$, velocity of contraction; $\mathrm{V}_{1 \mathrm{so}}$, isolength velocity of myocardial shortening.
} 
such inotropic agents as cardiac glycosides (12). Thus, it is possible that surgical trauma, the open chest, and the administration of anesthestic agents, which alter the central nervous system's regulation of circulatory responses and depress the myocardial contractile state, may also modify the extent of carotid sinus regulation of cardiac contractility.

Accordingly, the present investigation was conducted in normal, healthy, conscious dogs instrumented for the continuous measurement of left ventricular (LV) pressure, dimensions, rate of change of pressure $(\mathrm{dP} / \mathrm{dt})$, velocity of myocardial shortening, and cardiac output. The goal of this study was to characterize the extent of carotid sinus reflex regulation of the myocardial contractile state in the normal, conscious animal. The effects of bilateral carotid artery occlusion (BCO) and bilateral CSNS were studied when heart rate was allowed to vary or was controlled, after autonomic blockade, and after the effects of carotid hypotension or nerve stimulation on peripheral resistance were minimized by the prior administration of phenoxybenzamine. Finally, the carotid sinus-induced effects on the LV contractile state were compared with those of pharmacologic agents which elevated or reduced systemic arterial pressure to similar levels as occurred with carotid occlusion and CSNS.

\section{METHODS}

10 mongrel dogs, weighing between 17 and $27 \mathrm{~kg}$ were anesthetized with $\mathrm{Na}$ pentobarbital, $30 \mathrm{mg} / \mathrm{kg}$. Through a thoracotomy in the fifth left intercostal space, miniature pressure gauges ${ }^{2}$ were implanted within the left ventricle through a stab wound in the apex; opposing ultrasonic diameter transducers ${ }^{8}$ were sutured to the epicardium of the anterior and posterior walls of the left ventricle and stimulator electrodes were sutured to the left atrium (10 dogs). Electromagnetic flow probes were placed around the aortic root (five dogs). Hydraulic cuff occluders were placed around both common carotid arteries (seven dogs) and were tested at operation to determine the volume of fluid necessary to inflate the cuffs sufficiently to occlude the vessel. Stimulating electrodes ${ }^{4}$ were implanted on the carotid sinus nerves (six dogs).

The LV pressure gauges (13), were calibrated in vivo against a calibrated Statham P23 Db strain gauge manometer. $^{5}$ At autopsy their position within the ventricular cavity was confirmed. A Medtronic pacemaker ${ }^{\star}$ was used for electrical stimulation of the atrium. Aortic blood flow was measured with an electromagnetic flowmeter (Statham SP 2300). ${ }^{\circ}$ An improved ultrasonic transit time dimension gauge was used to measure $\mathrm{LV}$ diameter $;^{\circ}$ its principle of operation is similar to that of other ultrasonic gauges described pre-

\footnotetext{
${ }^{2}$ Konigsberg P22, Konigsberg Instruments, Pasadena, Calif.

${ }^{3}$ Construction details available from authors.

${ }^{4}$ Medtronic, Inc., Minneapolis, Minn.

${ }^{5}$ Statham Instruments, Los Angeles, Calif.

${ }^{\circ}$ Circuit diagrams available from authors.
}

viously (14-16). This instrument measures the transit time of acoustic impulses traveling at the sonic velocity of approximately $1.5 \times 10^{6} \mathrm{~mm} / \mathrm{sec}$ between the $5 \mathrm{MHz}$ piezoelectric crystals sutured to the LV epicardium at opposing sites. It was calibrated by substituting signals of known time duration from a pulse generator which was referenced to a quartz crystal controlled oscillator frequency. A voltage proportional to transit time was recorded and calibrated in terms of crystal separation. In this manner a measure of the external diameter was continuously recorded. At a constant temperature the drift of the instrument is less than $0.15 \mathrm{~mm} / \mathrm{hr}$, and its frequency response is $0-60 \mathrm{~Hz}$.

The experiments were conducted 2 wk to 2 months postoperatively, when the dogs had recovered from operation and were again vigorous and healthy and the discomforting side effects of carotid occlusion sinus nerve stimulation, i.e. local somatic irritations, were minimal. While the dogs were resting quietly, control records of LV pressure $(\mathrm{P})$, diameter (D), the time rate of change of diameter $(\mathrm{dD} / \mathrm{dt})$, i.e. the velocity of myocardial shortening $(\mathrm{V}), \mathrm{dP} / \mathrm{dt}$, cardiac output, and heart rate were obtained. These variables were continuously recorded during all interventions.

The common carotid arteries were occluded for 30-60-sec periods by inflating the hydraulic cuffs. The experiment was excluded from analysis if the dog moved or showed any awareness of the occlusion. Terminally, the pressure in the carotid artery distal to the occlusion was sampled with a catheter and measured with a Statham P23 Db strain gauge manometer. ${ }^{5}$ A $60 \mathrm{sec}$ period of occlusion produced a sustained decrease in mean and phasic carotid artery pressures distal to the cuff. Mean arterial pressure distal to the carotid cuffs was decreased by an average of $38 \mathrm{~mm} \mathrm{Hg}$ during the steady-state pressor response. Bilateral CSNS was carried out with a device having a $0.3 \mathrm{msec}$ pulse, and a rectangular wave form, and a repetition rate of $50 \mathrm{pulses} / \mathrm{sec}$. (17). The amplitude was adjusted to between 2.5 and $5.0 \mathrm{v}$ for each dog at the beginning of the experimental day and was then held constant. The strength of stimulus was the highest that could be delivered without the dog presenting any evidence of being aware of stimulation.

The effects of carotid occlusion and CSNS were studied after carotid sinus nerve section; in the animals with stimulating electrodes the carotid sinus nerves were sectioned cephalad to the electrodes. After carotid sinus denervation neither occlusion nor stimulation produced any hemodynamic alteration.

The effects of carotid occlusion and of CSNS were also observed after beta adrenergic blockade with propranolol, $1.0 \mathrm{mg} / \mathrm{kg}$, cholinergic blockade with atropine, 0.1-0.2 $\mathrm{mg} / \mathrm{kg}$, and alpha adrenergic blockade with phenoxybenzamine, ${ }^{8} 10.0 \mathrm{mg} / \mathrm{kg}$. Atrial stimulation was employed to maintain heart rate constant during carotid occlusion, while heart rate spontaneously returned to control during CSNS. I.v. norepinephrine, $0.5-1.5 \mu \mathrm{g} / \mathrm{kg}$, and methoxamine, $40-300$ $\mu \mathrm{g} / \mathrm{kg}$, were used to elevate LV pressure to levels identical to those resulting from carotid occlusion, and the effects of elevating pressure by each of these two agents were compared with those produced by carotid occlusion. The dosage of these two drugs necessary to elevate pressure to the levels achieved during carotid occlusion varied among animals. The effects of the same dosages of these drugs were also examined after phenoxybenzamine. In addition, the dose of

\footnotetext{
${ }^{7}$ Supplied by Ayerst Laboratories, New York, N. Y.

${ }^{8}$ Supplied by Smith Kline \& French Laboratories, Philadelphia, $\mathrm{Pa}$.
} 


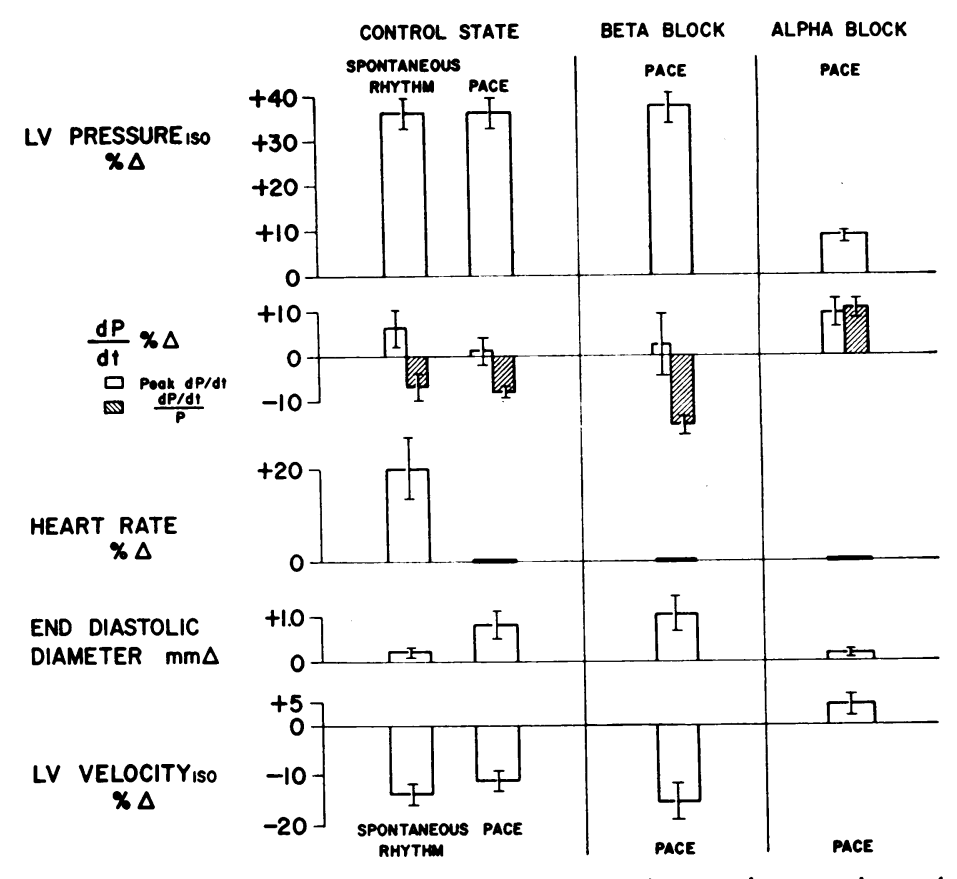

FIgURE 1 The average ( \pm SEM) percentage changes in a variety of circulatory measurements during the peak steady-state response to bilateral carotid artery occlusion. Changes in the control state are compared for seven dogs both when there was sinus rhythm, i.e. when heart rate was allowed to vary (left panel), and when heart rate was maintained constant with atrial stimulation; in five dogs after beta receptor blockade with propranolol and with heart rate constant (middle panel); and in six dogs after alpha receptor blockade with phenoxybenzamine and with heart rate constant (right panel).

norepinephrine which produced an inotropic response of comparable magnitude as that which occurred with carotid occlusion after alpha receptor blockade was determined. Nitroglycerin, $10-40 \mu \mathrm{g} / \mathrm{kg}$, was used to reduce LV systolic pressure to the same level obtained with CSNS and the effects on LV dynamics of these two methods of lowering arterial pressure were determined.

Data were recorded on a multichannel tape recorder and played back on a direct writing oscillograph at a paper speed of $100 \mathrm{~mm} / \mathrm{sec}$. The results of three periods of carotid occlusion and CSNS were averaged for each animal. A cardiotachometer triggered by the signal from the pressure pulse provided instantaneous and continuous records of heart. rate. An electronic RC filter was used to derive mean aortic blood flow, i.e., cardiac output. In addition the area of positive flow was integrated by planimetry to confirm stroke volume. Continuous records of $\mathrm{dP} / \mathrm{dt}$ and $\mathrm{dD} / \mathrm{dt}$ were derived from the $L V$ pressure and diameter signals using Philbrick $^{\ominus}$ operational amplifiers connected as differentiators. A triangular wave signal with known slope (rate-of-change) was substituted for pressure and diameter signals to calibrate directly the $\mathrm{dP} / \mathrm{dt}$ and $\mathrm{dD} / \mathrm{dt}$ channels.

The effects of carotid occlusion, CSNS, norepinephrine, methoxamine, and nitroglycerin on myocardial force-velocity relations were assessed by determining their effects on the velocity of shortening and intraventricular pressure $(P)$ at an identical ventricular diameter (D) by the technique de-

\footnotetext{
'Teledyne Philbrick Co., Dedham, Mass.
}

scribed in detail previously $(12,18)$. When, at any given instantaneous myocardial diameter or length (isolength point), the velocity of shortening ( $\left.\mathrm{V}_{1 \text { solength, i.e., }} \mathrm{V}_{1 \mathrm{so}}\right)$ increases, while intraventricular pressure $\left(\mathrm{P}_{1 \text { solength, }}\right.$ i.e., $\left.\mathrm{P}_{1 \mathrm{so}}\right)$ rises or remains constant, a shift in myocardial force-velocity relations reflecting a positive inotropic effect is considered to have occurred. All isolength points were obtained during the first one-third of ejection. In addition, the effects on peak $\mathrm{dP} / \mathrm{dt}$ and the quotient of $\mathrm{dP} / \mathrm{dt}$ and developed pressure, $P$ (left ventricular minus end-diastolic pressure), i.e. $(\mathrm{dP} / \mathrm{dt}) / \mathrm{P}$, were examined. The same level of pressure which occurred during isometric contraction, before and after each intervention, was used for this calculation and $\mathrm{dP} / \mathrm{dt}$ and $\mathrm{P}$ were determined at that level of pressure. This technique for evaluating the myocardial contractile state has been described in detail previously $(12,19,20)$.

\section{RESULTS}

\section{Bilateral carotid artery occlusion}

Control. In seven dogs studied in the control state, when heart rate was maintained constant, LV systolic pressure rose from $122 \pm 4$ to a steady-state level of $172 \pm 6 \mathrm{~mm} \mathrm{Hg}$, which occurred an average of $23 \pm 7$ SEM sec after the onset of carotid occlusion. At this time $P_{1 s o}$ had increased from an average of $120 \pm 4$ to $163 \pm 5 \mathrm{~mm} \mathrm{Hg}$ (Fig. 1, Table I) while LV end di- 
TABLE I

Effects of Carotid

\begin{tabular}{|c|c|c|c|c|}
\hline \multirow[b]{2}{*}{ Experiments } & \multicolumn{2}{|c|}{ Heart rate } & \multicolumn{2}{|c|}{ Pressure-peak systolic/systolic $\downarrow /$ end diastolic } \\
\hline & Control & $\begin{array}{l}\text { Carotid } \\
\text { occlusion* }\end{array}$ & Control & $\begin{array}{l}\text { Carotid } \\
\text { occlusion* }\end{array}$ \\
\hline & \multicolumn{2}{|c|}{ beats $/ \min$} & \multicolumn{2}{|c|}{$m m \mathrm{Hg}$} \\
\hline \multicolumn{5}{|l|}{ Experiment 1} \\
\hline Spontaneous rhythm & 84 & 90 & $109 / 106 / 6$ & $144 / 140 / 80$ \\
\hline Paced rhythm & 100 & 100 & $111 / 109 / 6$ & $143 / 138 / 8$ \\
\hline Beta block, paced & 100 & 100 & $111 / 109 / 7$ & $145 / 139 / 10$ \\
\hline Alpha block, paced & 120 & 120 & $97 / 94 / 4$ & $104 / 102 / 4$ \\
\hline \multicolumn{5}{|l|}{ Experiment 2} \\
\hline Spontaneous rhythm & 67 & 71 & $115 / 113 / 7$ & $165 / 154 / 9$ \\
\hline Paced rhythm & 100 & 100 & $114 / 110 / 6$ & $166 / 155 / 9$ \\
\hline Beta block, paced & 100 & 100 & $112 / 108 / 8$ & $161 / 150 / 10$ \\
\hline Alpha block, paced & 100 & 100 & $106 / 101 / 5$ & $116 / 113 / 5$ \\
\hline \multicolumn{5}{|l|}{ Experiment 3} \\
\hline Spontaneous rhythm & 61 & 69 & $131 / 128 / 8$ & $184 / 178 / 10$ \\
\hline Paced rhythm & 100 & 100 & $136 / 134 / 5$ & $182 / 176 / 8$ \\
\hline Beta block, paced & 100 & 100 & $134 / 131 / 7$ & $181 / 173 / 10$ \\
\hline Alpha block, paced & 100 & 100 & $125 / 123 / 4$ & $133 / 132 / 5$ \\
\hline \multicolumn{5}{|l|}{ Experiment 4} \\
\hline Spontaneous rhythm & 81 & 117 & $116 / 116 / 7$ & $188 / 176 / 9$ \\
\hline Paced rhythm & 120 & 120 & $118 / 118 / 5$ & $184 / 173 / 10$ \\
\hline Beta block, paced & 110 & 110 & $119 / 118 / 8$ & $179 / 170 / 11$ \\
\hline Alpha block, paced & 124 & 124 & $111 / 108 / 4$ & $120 / 118 / 4$ \\
\hline \multicolumn{5}{|l|}{ Experiment 5} \\
\hline Spontaneous rhythm & 89 & 124 & $135 / 129 / 7$ & $193 / 181 / 9$ \\
\hline Paced rhythm & 124 & 124 & $124 / 123 / 4$ & $173 / 173 / 9$ \\
\hline Beta block, paced & 120 & 120 & $130 / 124 / 7$ & $189 / 182 / 11$ \\
\hline Alpha block, paced & 120 & 120 & $107 / 104 / 5$ & $111 / 107 / 5$ \\
\hline \multicolumn{5}{|l|}{ Experiment 6} \\
\hline Spontaneous rhythm & 61 & 88 & $126 / 124 / 7$ & $170 / 161 / 9$ \\
\hline Paced rhythm & 100 & 100 & $126 / 124 / 4$ & $175 / 169 / 8$ \\
\hline Alpha block, paced & 100 & 100 & $108 / 106 / 4$ & $118 / 117 / 4$ \\
\hline \multicolumn{5}{|l|}{ Experiment 7} \\
\hline Spontaneous rhythm & 78 & 94 & $124 / 121 / 6$ & $161 / 153 / 7$ \\
\hline Paced rhythm & 120 & 120 & $128 / 124 / 4$ & $165 / 158 / 7$ \\
\hline \multicolumn{5}{|l|}{ Average \pm SEM } \\
\hline Spontaneous rhythm & $74 \pm 4$ & $95 \pm 8$ & $122 \pm 4 / 120 \pm 4 / 7 \pm 1$ & $172 \pm 5 / 164 \pm 5 / 9 \pm 1$ \\
\hline Paced rhythm & 109 & 109 & $122 \pm 4 / 120 \pm 4 / 5 \pm 1$ & $169 \pm 5 / 163 \pm 5 / 9 \pm 1$ \\
\hline Beta block, paced & 106 & 106 & $121 \pm 6 / 118 \pm 6 / 7 \pm 1$ & $171 \pm 9 / 163 \pm 8 / 10 \pm 1$ \\
\hline Alpha block, paced & 111 & 111 & $109 \pm 3 / 106 \pm 3 / 4 \pm 1$ & $117 \pm 4 / 115 \pm 4 / 5 \pm 1$ \\
\hline
\end{tabular}

* Peak steady-state results.

$\ddagger$ Isolength.

astolic pressure had risen from $5 \pm 1$ to $9 \pm 1 \mathrm{~mm} \mathrm{Hg}$. End diastolic diameter increased slightly, by $0.8 \pm 0.3$ $\mathrm{mm}$ from a control of $63.4 \pm 1.2 \mathrm{~mm}$, while end systolic diameter increased by $0.6 \pm 0.2 \mathrm{~mm}$ from a con- trol of $57.0 \pm 0.8 \mathrm{~mm}$. Viso decreased by $12 \pm 2 \%$ from a control of $60 \pm 2 \mathrm{~mm} / \mathrm{sec}$, as $\operatorname{did}(\mathrm{dP} / \mathrm{dt}) / \mathrm{P}$ by 8 $\pm 1 \%$, from a control of $44 \pm 2 \mathrm{sec}^{-1}$. Cardiac output and stroke volume showed little change, but peak aortic 
Occlusion

\begin{tabular}{|c|c|c|c|c|c|c|c|}
\hline \multicolumn{2}{|c|}{ Peak $d P / d t$} & \multicolumn{2}{|c|}{$(\mathrm{dP} / \mathrm{dt}) / \mathrm{P}$} & \multicolumn{2}{|c|}{ Diameter-end diastolic/end systolic } & \multicolumn{2}{|c|}{ Velocity $\ddagger$} \\
\hline Control & $\begin{array}{c}\text { Carotid } \\
\text { occlusion* }\end{array}$ & Control & $\begin{array}{l}\text { Carotid } \\
\text { occlusion* }\end{array}$ & Control & $\begin{array}{l}\text { Carotid } \\
\text { occlusion* }\end{array}$ & Control & $\begin{array}{l}\text { Carotid } \\
\text { occlusion* }\end{array}$ \\
\hline \multicolumn{2}{|c|}{$m m \mathrm{Hg} / \mathrm{sec}$} & \multicolumn{2}{|c|}{$\sec ^{-1}$} & \multicolumn{2}{|c|}{$m m$} & \multicolumn{2}{|c|}{$\mathrm{mm} / \mathrm{sec}$} \\
\hline 4070 & 3550 & 51 & 42 & $64.3 / 57.3$ & $64.3 / 57.4$ & 61 & 49 \\
\hline 4020 & 3690 & 50 & 46 & $63.9 / 57.4$ & $64.1 / 57.8$ & 57 & 50 \\
\hline 3540 & 2920 & 44 & 35 & $64.3 / 57.9$ & $64.6 / 58.2$ & 49 & 43 \\
\hline 3800 & 3990 & 46 & 51 & $59.8 / 56.3$ & $59.8 / 56.3$ & 49 & 50 \\
\hline 3940 & 3940 & 38 & 34 & $65.8 / 58.8$ & $66.0 / 59.0$ & 54 & 49 \\
\hline 4260 & 4260 & 39 & 35 & $63.9 / 57.6$ & $64.2 / 57.8$ & 53 & 48 \\
\hline 3540 & 3540 & 33 & 28 & $65.0 / 58.6$ & $65.4 / 58.8$ & 47 & 41 \\
\hline 3770 & 4060 & 34 & 37 & $62.2 / 56.2$ & $62.2 / 56.2$ & 44 & 44 \\
\hline 4410 & 4760 & 41 & 36 & $61.8 / 53.4$ & $62.2 / 54.1$ & 77 & 67 \\
\hline 4760 & 4760 & 44 & 39 & $58.2 / 53.4$ & $59.2 / 54.1$ & 68 & 62 \\
\hline 3530 & 3530 & 32 & 27 & $61.4 / 55.6$ & $62.4 / 56.3$ & 62 & 50 \\
\hline 3930 & 4400 & 36 & 40 & $56.9 / 52.0$ & $56.9 / 52.0$ & 62 & 62 \\
\hline 3330 & 4070 & 40 & 38 & $64.0 / 58.7$ & $64.2 / 59.0$ & 58 & 46 \\
\hline 3510 & 4280 & 42 & 40 & $60.8 / 55.6$ & $61.3 / 55.9$ & 58 & 47 \\
\hline 2670 & 3150 & 23 & 21 & $63.4 / 57.6$ & $63.7 / 51.9$ & 44 & 35 \\
\hline 3150 & 3710 & 38 & 45 & $60.0 / 55.8$ & $60.0 / 55.4$ & 47 & 52 \\
\hline 4330 & 4970 & 42 & 42 & $64.8 / 57.8$ & $65.0 / 58.8$ & 63 & 50 \\
\hline 4760 & 4760 & 39 & 37 & $62.6 / 56.6$ & $65.0 / 58.6$ & 58 & 50 \\
\hline 3470 & 3890 & 33 & 27 & $64.0 / 58.2$ & $66.9 / 59.2$ & 54 & 41 \\
\hline 3110 & 3450 & 30 & 33 & $61.1 / 57.6$ & $61.2 / 57.8$ & 44 & 49 \\
\hline 3480 & 3650 & 44 & 44 & $68.6 / 60.1$ & $68.6 / 60.3$ & 68 & 58 \\
\hline 3650 & 3480 & 46 & 42 & $67.0 / 59.3$ & $67.3 / 59.6$ & 68 & 63 \\
\hline 3190 & 3190 & 40 & 40 & $66.5 / 59.3$ & $66.5 / 59.3$ & 65 & 65 \\
\hline 3330 & 3500 & 42 & 40 & $68.2 / 59.0$ & $68.2 / 59.5$ & 53 & 49 \\
\hline 3660 & 3660 & 46 & 41 & $67.4 / 59.0$ & $68.0 / 59.8$ & 59 & 49 \\
\hline $3740 \pm 410$ & $4070 \pm 460$ & $44 \pm 2$ & $40 \pm 2$ & $65.4 \pm .9 / 57.9 \pm 0.8$ & $65.7 \pm .9 / 58.3 \pm 0.8$ & $62 \pm 3$ & $53 \pm 3$ \\
\hline $4090 \pm 210$ & $4130 \pm 190$ & $44 \pm 2$ & $40 \pm 2$ & $63.4 \pm 1.2 / 51.0 \pm 0.8$ & $64.2 \pm 1.2 / 57.6 \pm 0.8$ & $60 \pm 2$ & $53 \pm 3$ \\
\hline $3380 \pm 100$ & $3400 \pm 140$ & $33 \pm 2$ & $28 \pm 2$ & $63.6 \pm .6 / 57.6 \pm 0.5$ & $64.8 \pm .8 / 58.1 \pm 0.5$ & $51 \pm 2$ & $42 \pm 2$ \\
\hline $3490 \pm 160$ & $3800 \pm 160$ & $37 \pm 2$ & $41 \pm 2$ & $61.1 \pm 1.3 / 56.2 \pm 1.0$ & $61.1 \pm 1.3 / 56.2 \pm 1.0$ & $52 \pm 4$ & $54 \pm 4$ \\
\hline
\end{tabular}

flow velocity decreased by $16 \pm 3 \%$ from a control of 12.7 liters/min (Fig. 2).

When heart rate was allowed to vary in these 7 dogs, the maximal increase in heart rate generally occurred within the first $5 \mathrm{sec}$ of carotid occlusion and during the steady state of the maximal pressor effect, heart rate had returned toward control but still remained elevated by $22 \pm 7 \%$ above a control of $74 \pm 4$ beats/ 

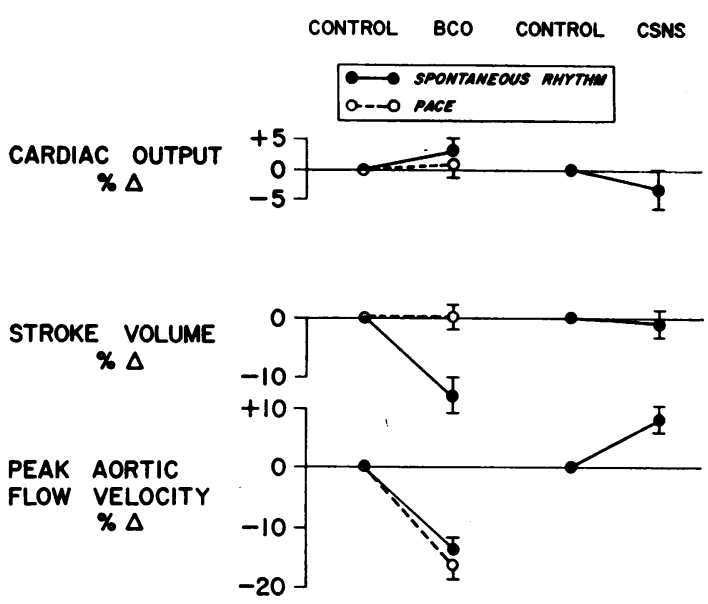

FIGURE 2 The average ( $\pm_{\text {SEMF }}$ ) percentage change in cardiac output, stroke volume, and peak aortic flow velocity to carotid occlusion (BCO) both when heart rate was maintained constant (broken line) and when it was allowed to vary (solid line) (left panel) and to carotid sinus nerve stimulation (right panel). min (Fig. 1, Table I). When heart rate was permitted to rise, $\mathrm{BCO}$ increased $\mathrm{P}_{\mathbf{1 s o}}$ and decreased $\mathrm{V}_{\text {iso }}$ and $(\mathrm{dP} / \mathrm{dt}) / \mathrm{P}$ to levels which were comparable to those noted when heart rate was maintained constant (Fig. 1, Table I), but the increase in end diastolic diameter $(0.2$ $\pm 0.1 \mathrm{~mm}$ ) was less and while cardiac output again remained constant, stroke volume decreased by $13 \pm 2 \%$ (Figs. 2, 3). Thus, in the healthy, conscious dog when heart rate was allowed to vary or was held constant, BCO increased systolic pressure significantly but produced little detectable alteration in the contractile state.

Beta receptor blockade. In five dogs, propranolol, $1.0 \mathrm{mg} / \mathrm{kg}$, caused slight increases in end diastolic pressure and diameter and end systolic diameter and slight decreases in $\mathrm{V}_{1 \mathrm{so}}$ and $(\mathrm{dP} / \mathrm{dt}) / \mathrm{P}$ but did not significantly affect LV systolic pressure or heart rate. In this situation, with heart rate held constant by means of atrial stimulation, BCO caused similar increases in $P_{\mathbf{s} s}$, end diastolic and end systolic diameters and end diastolic pressure (Fig. 1, Table I). The reductions in

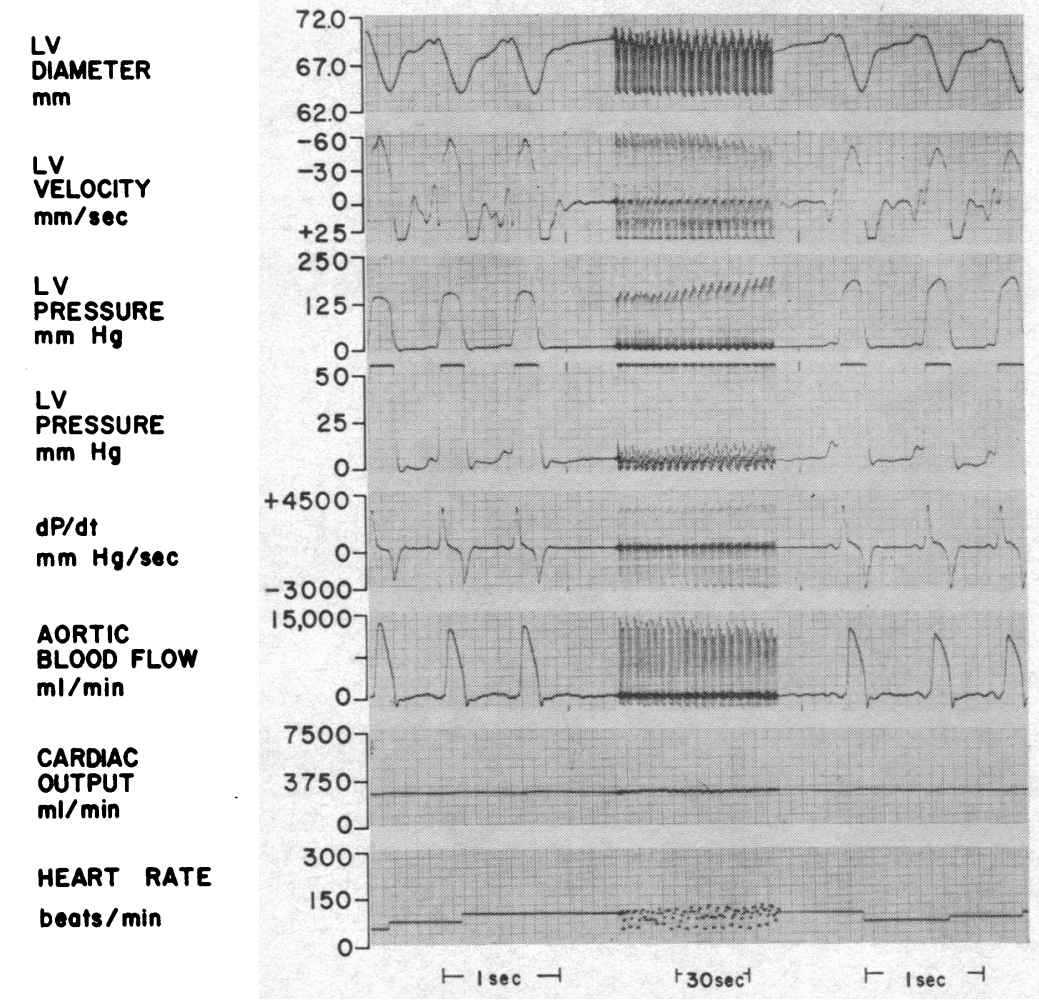

FIGURE 3 A typical record of a response to $\mathrm{BCO}$ in one of the experimental animals in the control state. The instantaneous record of LV diameter, velocity, systolic pressure, diastolic pressure, $\mathrm{dP} / \mathrm{dt}$, aortic flow velocity, cardiac output, and heart rate are shown at fast and slow paper speeds. In this case heart rate was allowed to vary and only a slight tachycardia resulted from $\mathrm{BCO}$. The sinus arrhythmia is characteristic of the healthy, unsedated, conscious dog. 
Viso (Fig. 4) and (dP/dt)/P (Fig. 5), were similar to those which were noted in the control state. Thus, beta receptor blockade had little influence on the response to $\mathrm{BCO}$, indicating that beta receptor activation in the myocardium is not an important feature of the response to carotid occlusion.

Alpha adrenergic blockade. In six dogs, in order to separate the effects of $\mathrm{BCO}$ on LV contractile function from those associated with the induced change in systemic arterial pressure, phenoxybenzamine was given. This drug had a slight negative intotropic effect; it lowered LV systolic and diastolic pressures and diameters, $\mathrm{V}_{\text {iso, and }}(\mathrm{dP} / \mathrm{dt}) / \mathrm{P}$. Under these circumstances, and with heart rate maintained constant, $\mathrm{BCO}$ did not alter end diastolic pressure or diameter or end systolic diameter, and $\mathrm{P}_{\mathrm{iso}}$ increased only slightly but significantly $(P<0.01)$, by $8 \pm 1 \%$ from a postphenoxybenzamine control of $106 \pm 3 \mathrm{~mm} \mathrm{Hg}$ (Fig. 1, Table I). In contrast to the results in the control state or after beta receptor blockade, BCO now slightly increased $\mathrm{V}_{\text {iso }}$ by $4 \pm 2 \%(P<0.01)$ (Fig. 4$)$ from a control of $52 \pm 4 \mathrm{~mm} / \mathrm{sec}$ and increased $(\mathrm{dP} / \mathrm{dt}) / \mathrm{P}$ by $10 \pm 2 \%$ $\left(P<0.01\right.$ ), from a control of $37 \pm 2 \mathrm{sec}^{-1}$ (Fig. 5). Thus, when alpha blockade limited the changes in peripheral resistance, $\mathrm{BCO}$ produced a minor increase in the contractile state. However, this slight change failed to increase contractility even to the level which existed before phenoxybenzamine administration.

Cholinergic blockade. After atropine $(0.1 \mathrm{mg} / \mathrm{kg})$ in three dogs with heart rate maintained constant, $\mathrm{BCO}$ produced effects similar to those observed in the dogs in the control state; $\mathrm{P}_{\mathrm{tso}}$ increased by $36 \%$ from an average of $117 \mathrm{~mm} \mathrm{Hg}$, end diastolic diameter increased by $0.9 \mathrm{~mm}$, while $\mathrm{V}_{\text {iso }}$ decreased by $14 \%$ from $58 \mathrm{~mm} /$ sec and $(\mathrm{dP} / \mathrm{dt}) / \mathrm{P}$ decreased by $10 \%$ from an average of $42 \mathrm{sec}^{-1}$. Thus, parasympathetic activation probably played little role in the response to BCO.

Responses to methoxamine and norepinephrine (seven dogs). Control levels before drug administration were similar to those before BCO. In all seven dogs when $\mathrm{LV}$ systolic pressure was raised with methoxamine to the level attained during $\mathrm{BCO}$, changes similar to those which occurred during $\mathrm{BCO}$ were noted, i.e. $\mathrm{P}_{\mathrm{ts}}$ rose,

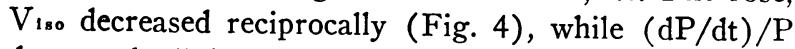
decreased slightly (Fig. 5). After phenoxybenzamine a similar dose of methoxamine (average dose $=200$ $\mu \mathrm{g} / \mathrm{kg}$ ) had only a negligible effect on $\mathrm{P}_{\mathrm{lso}}$, and no detectable effect on Viso (Fig. 4). Thus, methoxamine, a drug which does not exert a direct myocardial effect and elevates arterial pressure by systemic vasoconstriction, produced almost no detectable inotropic response, an effect almost indistinguishable from that of carotid occlusion.

In contrast to the responses to $\mathrm{BCO}$ and methox-

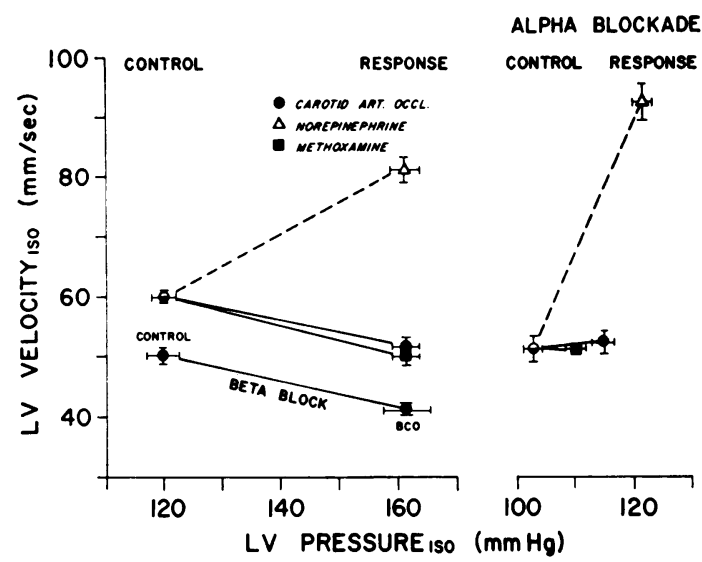

FIGURE 4 Average ( $\pm_{\text {SEM }}$ ) pressure-velocity relations for the responses to $\mathrm{BCO}$, norepinephrine, and methoxamine in the control state shown in the left panel, and after phenoxybenzamire, shown in the right panel. The response to $\mathrm{BCO}$ after beta receptor blockade is shown on the left but is slightly below the other responses. All pressure and velocity measurements in the same dog were made at a constant length both during control and response, and the changes thereafter reflect changes in the force-velocity relation (12, $18,23)$.

amine, norepinephrine, an agent known to elevate arterial pressure but also to stimulate myocardial contractility through its action on beta adrenergic receptors, in an average dose of $1.25 \mu \mathrm{g} / \mathrm{kg}$, while exerting a similar pressor action, substantially increased $\mathrm{V}_{\mathrm{iso}}$ by $36 \pm 5 \%$, while raising $\mathrm{P}_{1 \text { so }}$ by $36 \pm 3 \%$ (Fig. 4) and $(\mathrm{dP} / \mathrm{dt}) / \mathrm{P}$ by $56 \pm 7 \%$ (Fig. 5 ). After phenoxybenzamine the same dose of norepinephrine increased $\mathrm{P}_{\mathrm{iso}}$ by only $19 \pm 4 \%$ but increased $V_{1 \text { so }}$ by $81 \pm 9 \%$ and $(\mathrm{dP} / \mathrm{dt}) / \mathrm{P}$ by $118 \pm 12 \%$. Thus, norepinephrine in contrast to $\mathrm{BCO}$ and methoxamine produced a powerful inotropic response. A much smaller dose of norepinephrine, $0.01-0.02 \mu \mathrm{g} / \mathrm{kg}$, after phenoxybenzamine,

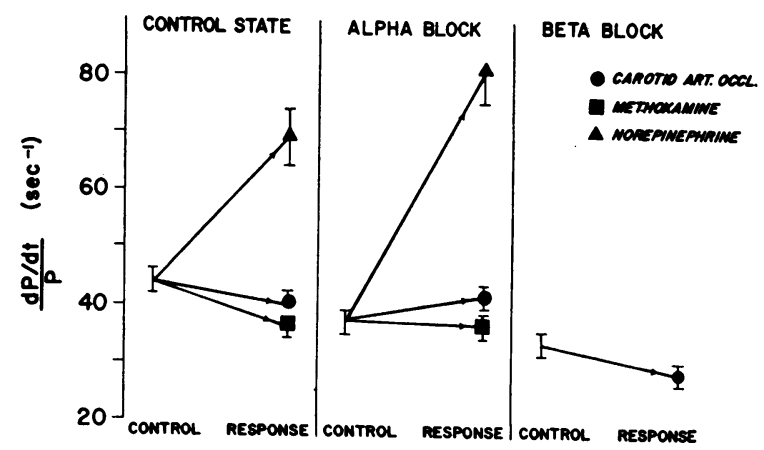

Figure 5 Average ( \pm SEM) values for $(\mathrm{dP} / \mathrm{dt}) / \mathrm{P}$, an index of myocardial contractility $(12,19,20)$ during control and response to $\mathrm{BCO}$, norepinephrine and methoxamine in the control state (left panel), after alpha blockade (middle panel), and the response to BCO after heta receptor blockade (right panel).

Carotid Sinus Control of Contractility 
TABLE II

\begin{tabular}{|c|c|c|c|c|c|c|}
\hline \multirow[b]{2}{*}{ Experiments } & \multicolumn{2}{|c|}{ Heart rate } & \multicolumn{2}{|c|}{ Pressure—-peak systolic/systolicł/end diastolic } & \multicolumn{2}{|c|}{ Peak dP/dt } \\
\hline & Control & CSNS* & Control & CSNS* & Control & CSNS \\
\hline Experiment 1 & \multicolumn{2}{|c|}{ beals/min } & \multicolumn{2}{|c|}{$m m \mathrm{Hg}$} & \multicolumn{2}{|c|}{$m m \mathrm{Hg} / \mathrm{sec}$} \\
\hline $\begin{array}{l}\text { Control state } \\
\text { Beta block } \\
\text { Alpha block }\end{array}$ & $\begin{array}{r}89 \\
87 \\
111\end{array}$ & $\begin{array}{r}87 \\
85 \\
108\end{array}$ & $\begin{array}{c}119 / 116 / 6 \\
120 / 118 / 8 \\
99 / 96 / 4\end{array}$ & $\begin{array}{r}121 / 99 / 5 \\
100 / 98 / 6 \\
97 / 94 / 4\end{array}$ & $\begin{array}{l}4060 \\
3460 \\
3880\end{array}$ & $\begin{array}{l}3320 \\
3250 \\
3700\end{array}$ \\
\hline \multicolumn{7}{|l|}{ Experiment 2} \\
\hline $\begin{array}{l}\text { Control state } \\
\text { Beta block } \\
\text { Alpha block }\end{array}$ & $\begin{array}{r}64 \\
61 \\
106\end{array}$ & $\begin{array}{r}64 \\
61 \\
101\end{array}$ & $\begin{array}{l}125 / 123 / 8 \\
125 / 123 / 10 \\
115 / 113 / 5\end{array}$ & $\begin{array}{l}103 / 101 / 6 \\
104 / 102 / 8 \\
110 / 108 / 5\end{array}$ & $\begin{array}{l}3850 \\
3110 \\
3660\end{array}$ & $\begin{array}{l}3200 \\
2940 \\
3420\end{array}$ \\
\hline \multicolumn{7}{|l|}{ Experiment 3} \\
\hline $\begin{array}{l}\text { Control state } \\
\text { Beta block } \\
\text { Alpha block }\end{array}$ & $\begin{array}{r}82 \\
76 \\
116\end{array}$ & $\begin{array}{r}85 \\
75 \\
114\end{array}$ & $\begin{array}{l}122 / 121 / 7 \\
123 / 119 / 8 \\
116 / 114 / 5\end{array}$ & $\begin{array}{l}108 / 105 / 6 \\
107 / 104 / 6 \\
112 / 111 / 5\end{array}$ & $\begin{array}{l}3700 \\
3140 \\
3480\end{array}$ & $\begin{array}{l}3380 \\
3010 \\
3380\end{array}$ \\
\hline \multicolumn{7}{|l|}{ Experiment 4} \\
\hline $\begin{array}{l}\text { Control state } \\
\text { Beta block } \\
\text { Alpha block }\end{array}$ & $\begin{array}{r}78 \\
76 \\
112\end{array}$ & $\begin{array}{r}76 \\
74 \\
109\end{array}$ & $\begin{array}{l}124 / 120 / 7 \\
122 / 118 / 8 \\
112 / 112 / 5\end{array}$ & $\begin{array}{c}100 / 97 / 6 \\
99 / 96 / 8 \\
109 / 109 / 5\end{array}$ & $\begin{array}{l}4240 \\
3350 \\
3440\end{array}$ & $\begin{array}{l}4070 \\
3120 \\
3160\end{array}$ \\
\hline \multicolumn{7}{|l|}{ Experiment 5} \\
\hline $\begin{array}{l}\text { Control state } \\
\text { Alpha block }\end{array}$ & $\begin{array}{l}102 \\
119\end{array}$ & $\begin{array}{r}97 \\
111\end{array}$ & $\begin{array}{l}130 / 127 / 7 \\
107 / 104 / 4\end{array}$ & $\begin{array}{l}111 / 109 / 7 \\
101 / 100 / 4\end{array}$ & $\begin{array}{l}3740 \\
3270\end{array}$ & $\begin{array}{l}3160 \\
2910\end{array}$ \\
\hline \multicolumn{7}{|l|}{ Experiment 6} \\
\hline Control state & 86 & 84 & $119 / 115 / 8$ & $107 / 104 / 7$ & 3230 & 2980 \\
\hline \multicolumn{7}{|l|}{ Average \pm SEM } \\
\hline $\begin{array}{l}\text { Control state } \\
\text { Beta block } \\
\text { Alpha block }\end{array}$ & $\begin{array}{r}84 \pm 6 \\
75 \pm 6 \\
113 \pm 5\end{array}$ & $\begin{array}{r}83 \pm 6 \\
74 \pm 6 \\
109 \pm 5\end{array}$ & $\begin{array}{l}123 \pm 3 / 120 \pm 3 / 7 \pm 1 \\
123 \pm 3 / 119 \pm 3 / 9 \pm 1 \\
110 \pm 3 / 108 \pm 3 / 5 \pm 1\end{array}$ & $\begin{array}{l}108 \pm 2 / 105 \pm 2 / 6 \pm 1 \\
103 \pm 2 / 100 \pm 2 / 7 \pm 1 \\
106 \pm 2 / 104 \pm 2 / 5 \pm 1\end{array}$ & $\begin{array}{l}3800 \pm 180 \\
3270 \pm 90 \\
3550 \pm 100\end{array}$ & $\begin{array}{l}3350 \pm 170 \\
3100 \pm 40 \\
3310 \pm 140\end{array}$ \\
\hline
\end{tabular}

* Peak steady-state results.

‡ Isolength.

was found to produce an inotropic response equivalent to the small one produced by $\mathrm{BCO}$ after alpha blockade, in terms of the force-velocity and $(\mathrm{dP} / \mathrm{dt}) / \mathrm{P}$ relationships, the latter parameter rising by an average of $12 \pm 2 \%$.

\section{Carotid sinus nerve stimulation}

Control. In six dogs studied in the conscious state, CSNS resulted in a prompt reduction in heart rate which returned to control levels during the time of maximal pressure reduction, i.e. $15-20 \mathrm{sec}$ after the onset of stimulation, as has been noted in detail previously $(11,21,22)$. Peak LV systolic pressure declined from $125 \pm 4$ to $108 \pm 3 \mathrm{~mm} \mathrm{Hg}$. $P_{1 \text { во }}$ decreased by an average maximum of $15 \pm 2 \%$ from a control of $120 \pm 3$ $\mathrm{mm} \mathrm{Hg}$ (Fig. 6, Table II). End diastolic diameter de- creased slightly by $0.4 \pm 1 \mathrm{~mm}$ from a control of 67.0 $\pm 1.4 \mathrm{~mm}$, and end systolic diameter remained essentially unchanged. $\mathrm{LV}$ end diastolic pressure decreased from $7 \pm 1 \mathrm{~mm} \mathrm{Hg}$ to $6 \pm 1 \mathrm{~mm} \mathrm{Hg}$. $\mathrm{V}_{1 \mathrm{so}}$ increased slightly, by $11 \pm 2 \%$ from a control of $65 \pm 2 \mathrm{~mm} / \mathrm{sec}$ (Fig. 7), while (dP/dt)/P showed little change, 43 \pm 1 to $42 \pm 1 \sec ^{-1}$ (Fig. 8). The small reductions in

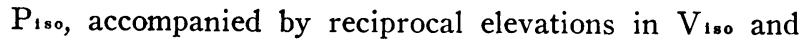
little change in $(\mathrm{dP} / \mathrm{dt}) / \mathrm{P}$, indicate that CSNS produced no significant change in the inotropic state. Cardiac output and stroke volume remained essentially constant, while peak aortic flow velocity increased by $8 \pm 2 \%$ (Fig. 2). Thus, CSNS, like BCO, altered systemic arterial resistance, and therefore $\mathrm{LV}$ systolic pressure, but produced little detectable effect on contractility. 
Sinus Nerve Stimulation

\begin{tabular}{|c|c|c|c|c|c|}
\hline \multicolumn{2}{|c|}{$(d P / d t) / P$} & \multicolumn{2}{|c|}{ Diameter-end diastolic/end systolic } & \multicolumn{2}{|c|}{ Velocitył } \\
\hline Control & CSNS* & Control & CSNS* & Control & CSNS* \\
\hline \multicolumn{2}{|c|}{$\sec ^{-1}$} & \multicolumn{2}{|c|}{$m m$} & \multicolumn{2}{|c|}{$\mathrm{mm} / \mathrm{sec}$} \\
\hline 46 & 45 & $72.2 / 63.9$ & $71.9 / 63.9$ & 64 & 67 \\
\hline 40 & 40 & $73.4 / 64.9$ & $73.0 / 64.7$ & 55 & 61 \\
\hline 43 & 40 & $69.3 / 64.3$ & $69.3 / 64.3$ & 64 & 61 \\
\hline 42 & 40 & $62.0 / 56.4$ & $61.2 / 55.6$ & 57 & 62 \\
\hline 38 & 38 & $63.0 / 58.2$ & $62.4 / 57.0$ & 52 & 59 \\
\hline 39 & 36 & $59.8 / 55.9$ & $59.8 / 55.9$ & 47 & 46 \\
\hline 44 & 44 & $67.5 / 59.2$ & $67.2 / 59.2$ & 74 & 84 \\
\hline 39 & 38 & $67.8 / 59.5$ & $67.5 / 59.2$ & 65 & 74 \\
\hline 41 & 40 & $65.9 / 59.2$ & $65.9 / 59.2$ & 60 & 58 \\
\hline 49 & 48 & $68.6 / 60.2$ & $68.4 / 60.2$ & 62 & 69 \\
\hline 32 & 32 & $69.2 / 60.9$ & $69.0 / 60.7$ & 49 & 58 \\
\hline 33 & 30 & $65.4 / 59.8$ & $65.3 / 59.1$ & 52 & 48 \\
\hline 40 & 37 & $65.5 / 59.5$ & $65.2 / 59.5$ & 63 & 69 \\
\hline 35 & 31 & $62.2 / 59.0$ & $62.3 / 59.1$ & 51 & 47 \\
\hline 38 & 38 & $66.4 / 58.3$ & $66.2 / 58.2$ & 68 & 75 \\
\hline $43 \pm 1$ & $42 \pm 1$ & $67.0 \pm 1.4 / 59.6 \pm 1.2$ & $66.7 \pm 1.5 / 59.4 \pm 1.3$ & $65 \pm 2$ & $72 \pm 3$ \\
\hline $37 \pm 2$ & $37 \pm 2$ & $68.4 \pm 1.7 / 60.9 \pm 1.5$ & $68.0 \pm 2.2 / 60.4 \pm 1.6$ & $55 \pm 2$ & $63 \pm 3$ \\
\hline $38 \pm 2$ & $35 \pm 2$ & $64.5 \pm 1.6 / 59.6 \pm 1.3$ & $64.5 \pm 1.6 / 59.5 \pm 1.3$ & $55 \pm 3$ & $52 \pm 3$ \\
\hline
\end{tabular}

Beta receptor blockade. In four dogs after propranolol, $1.0 \mathrm{mg} / \mathrm{kg}$, CSNS produced similar changes in ventricular diameter as in the control state. Also a reduction in $P_{1: 0}$ (average $=-16 \pm 1 \%$ ) was associated with a similar increase in $\mathrm{V}_{\mathrm{iso}}, 14 \pm 1 \%$, while $(\mathrm{dP} / \mathrm{dt}) / \mathrm{P}$ remained constant at $37 \pm 2 \mathrm{sec}^{-1}$ (Figs. $6-8)$. Thus, the similarity of the results of CSNS before and after beta blockade indicates that withdrawal of cardiac sympathetic tone does not contribute significantly to the response to CSNS in the control state.

Alpha adrenergic blockade. In five dogs, after phenoxybenzamine $(10.0 \mathrm{mg} / \mathrm{kg})$, CSNS did not alter ventricular diameter or reduce $\mathrm{P}_{\mathrm{iso}}$. Under these conditions, $V_{1 \text { so }}$ fell slightly, by $5 \pm 1 \%$ from a control of $55 \pm 3 \mathrm{~mm} / \mathrm{sec}$, and $(\mathrm{dP} / \mathrm{dt}) / \mathrm{P}$ decreased by $7 \pm 1 \%$ from a control of $38 \pm 2 \sec ^{-1}$ (Figs. 6, 8). These changes are consistent with a slight depression of the inotropic state. Thus, the relatively minor negative inotropic effects of CSNS were only apparent after the effects on peripheral resistance had been prevented by alpha blockade.

Nitroglycerin. In order to determine the effects on $\mathrm{LV}$ dynamics of the reduction of $\mathrm{LV}$ systolic pressure which occurred with CSNS, nitroglycerin was administered to six dogs. When LV systolic pressure decreased to the same level that occurred with CSNS and with heart rate maintained constant, $P_{1 \text { so }}$ fell by $15 \pm 2 \%$, $V_{1 s o}$ increased reciprocally by $14 \pm 3 \%$, while (dP/ $\mathrm{dt}) / \mathrm{P}$ remained essentially constant (Figs. 7,8 ). Thus, nitroglycerin, an agent which reduces arterial pressure 


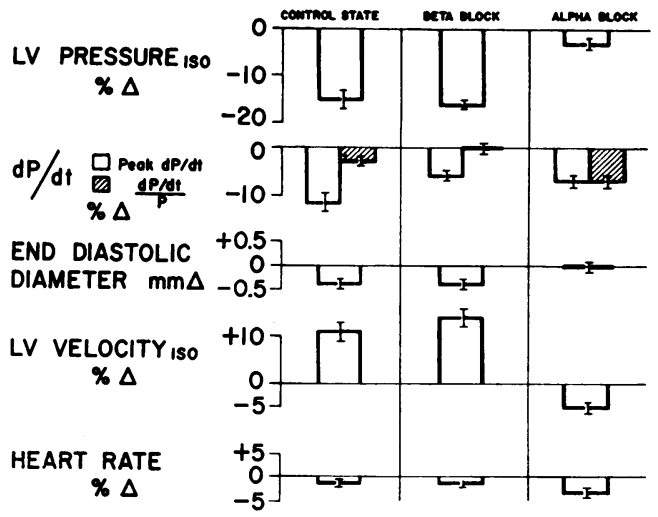

FIGURE 6 The average ( $\pm_{\mathrm{SEM}}$ ) percentage changes of a variety of measurements during the steady-state maximal depressor response to CSNS. Changes are compared during the control state in six dogs (left panel), after beta receptor blockade in four dogs (middle panel), and after alpha blockade five dogs (right panel).

by lowering systemic resistance and which is practically devoid of inotropic action, produced a similar response to that of CSNS.

Cholinergic blockade. After atropine, $0.2 \mathrm{mg} / \mathrm{kg}$, was administered to three dogs, CSNS produced similar results as in the control state; $P_{1 s o}$ decreased by $13 \%$, end diastolic diameter decreased by $0.2 \mathrm{~mm}$, while

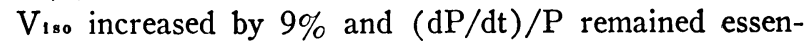
tially constant. Thus, parasympathetically mediated effects did not contribute significantly to the response to CSNS in the conscious dog.

Anesthesia and hemorrhage. In three dogs after pentobarbital $\mathrm{Na}, 30 \mathrm{mg} / \mathrm{kg}$, and with respiration controlled, CSNS decreased $P_{\text {iso }}$ slightly less than in the control state $(12 \%)$ but in contrast to the conscious

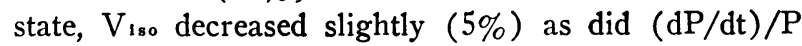

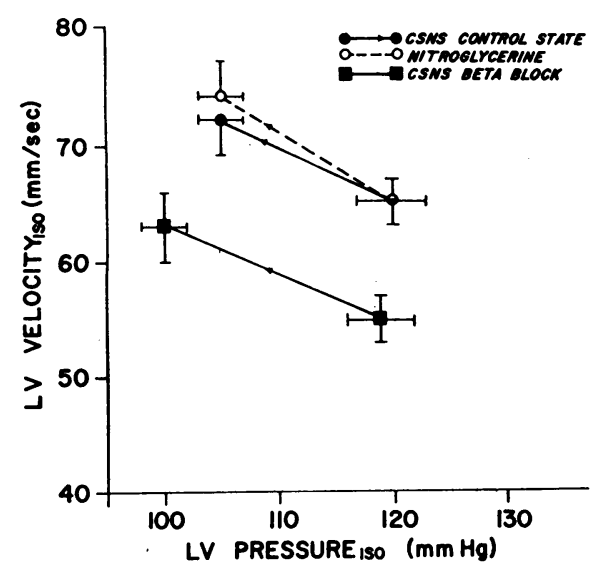

Figure 7 Average ( \pm SEM) pressure-velocity relations for the response to CSNS in the control state, to nitroglycerin in the control state, and to CSNS after beta receptor blockade.

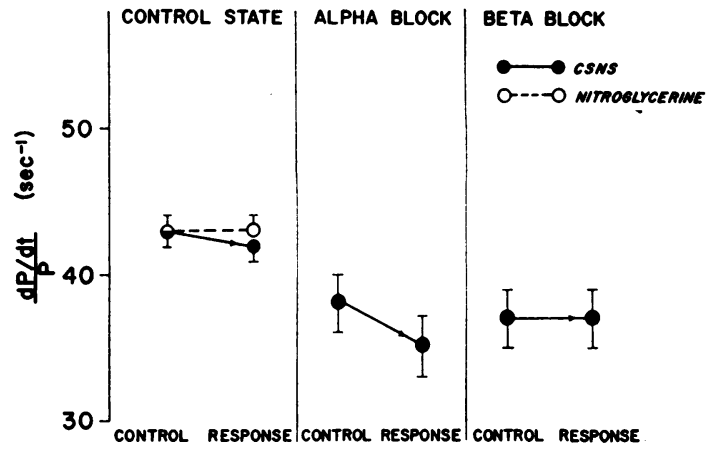

Figure 8 Average ( \pm SEM) values for $(d P / d t) / P$ before and during CSNS and nitroglycerin in the control state (left panel), the response to CSNS after alpha blockade (middle panel), and the response to CSNS after beta receptor blockade (right panel).

$(15 \%)$ (Figs. 8, 9), indicating that in the anesthetized state CSNS did depress contractility. After $500 \mathrm{~cm}^{3}$ of blood had been removed from each of the three anesthetized dogs, CSNS again reduced $\mathrm{P}_{\text {iso }}$ by $10 \%$, but now decreased $\mathrm{V}_{\text {iso }}(15 \%)$ and $(\mathrm{dP} / \mathrm{dt}) / \mathrm{P}(32 \%)$, to a greater extent than in the anesthetized state before blood loss (Figs. 8, 9).
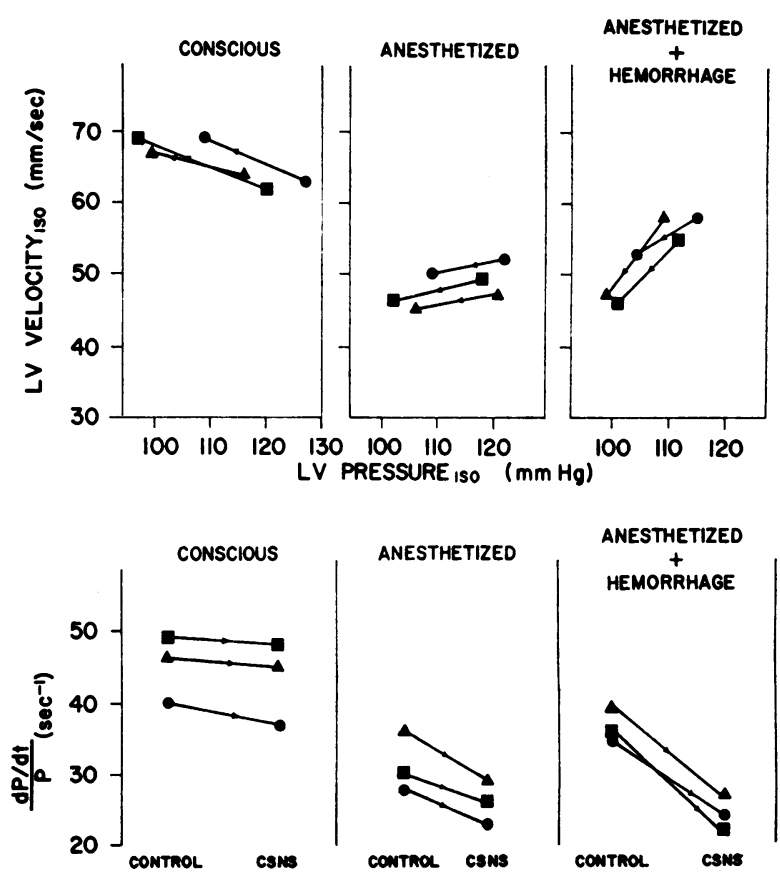

FIgURE 9 The individual responses to CSNS of three dogs represented by the separate symbols studied in the conscious control state (left panel), after anesthesia with $\mathrm{Na}$ pentobarbital (middle panel), and after anesthesia plus hemorrhage (right panel). The changes in force-velocity relations are depicted in the upper graphs and changes in $(\mathrm{dP} / \mathrm{dt}) / \mathrm{P}$ are shown in the lower graphs. 


\section{DISCUSSION}

The present investigation was conducted in intact unanesthetized dogs instrumented for direct and continuous measurements to evaluate the force-velocity-length and $(\mathrm{dP} / \mathrm{dt}) / \mathrm{P}$ relationships, which are currently accepted indicators of the myocardial contractile state $(12,19,20,23)$. The classical studies of A. V. Hill demonstrated that the inverse relationship between the force generated and the velocity of shortening is an important property of skeletal muscle (24). This relationship has subsequently been extended to the isolated papillary muscle preparation $(25,26)$ and this technique has been further extended to studies in the intact ventricle of anesthetized dogs $(27,28)$. It has also been utilized to characterize the myocardial contractile state in intact, unanesthetized man (18). Similarly, $\mathrm{dP} / \mathrm{dt}$ has been employed as an indicator of myocardial contractility and the relationship between $\mathrm{dP} / \mathrm{dt}$ and developed pressure $(\mathrm{dP} / \mathrm{dt}) / \mathrm{P}$ has recently been demonstrated to be a more useful index since it is relatively insentive to changes in preload and afterload $(19,20)$. Both of these indices (force-velocity relation and (dP) $\mathrm{dt}) / \mathrm{P}$ ) have been demonstrated to be useful in characterizing inotropic effects in the conscious animal (12) and accordingly were employed in this study to reflect induced changes in the myocardial contractile state. These indices are sensitive enough to reflect positive inotropic changes when agents known to increase contractility such as norepinephrine (Figs. 4,5 ) and cardiac glycosides are administered (12), and they reflect well the negative inotropic effect produced by agents known to depress the contractile state, i.e., propranolol (Figs. 4, 5) (12) and pentobarbital anesthesia (Fig. 9) (12).

Previous studies conducted in open-chest anesthetized animal preparations indicated that the regulation of myocardial contractility is one of the important functions of the carotid sinus reflex (1-8). In the present investigation conducted in normal, healthy, conscious dogs, the extent of control of the myocardial contractile state by the carotid sinuses was determined to be relatively minor. Bilateral common carotid artery occlusion substantially increased LV pressure and produced a reciprocal reduction in the velocity of LV shortening, and in the peak aortic ejection velocity, but did not alter $(\mathrm{dP} / \mathrm{dt}) / \mathrm{P}$ or cardiac output. This effect on the force-velocity relationship and $(\mathrm{dP} / \mathrm{dt}) / \mathrm{P}$ appeared similar to responses predicted from interventions which primarily increase afterload without having direct inotropic action $(18,19,23,28)$. Had carotid hypotension produced the expected augmentation of contractility, along with the rise in ventricular pressure, velocity of shortening would also have risen, or at least would have remained constant, while $(\mathrm{dP} / \mathrm{dt}) / \mathrm{P}$ would have risen.

To test the hypothesis that carotid occlusion produces alterations primarily in peripheral resistance while having little effect on the myocardial contractile state, the response to this stimulus was compared with that produced by equal pressor responses of norepinephrine, a drug that possesses both strong inotropic and vasoconstrictor properties, and of methoxamine, a drug that has virtually no inotropic action but raises pressure almost entirely by increasing peripheral resistance. Norepinephrine demonstrated classical iontropic properties; while increasing pressure to the same level as attained by carotid occlusion, it substantially augmented velocity $(36 \%)$ and $(\mathrm{dP} / \mathrm{dt}) / \mathrm{P}(56 \%)$, i.e., the effect anticipated had carotid occlusion resulted not only in systemic vasoconstriction but improvement in myocardial contractility as well. This finding demonstrates that the technique employed in this study is clearly capable of recognizing an improvement of the contractile state in the conscious dog in the face of vasoconstriction. On the other hand, methoxamine, a drug without inotropic action, produced a response very similar to that of carotid occlusion; the increase in pressure being accompanied by a reduction in velocity (Fig. 4) and of little change in $(\mathrm{dP} / \mathrm{dt}) / \mathrm{P}$ (Fig. 5). Thus, the similarity in responses to carotid occlusion and methoxamine and the dissimilarity between the responses to carotid occlusion and norepinephrine supports the hypothesis that carotid sinus hypotension has little inotropic effect in the conscious dog and that its main circulatory action is to elevate systemic vascular resistance.

Further evidence for this concept was derived from the results after beta receptor blockade. If the response to carotid occlusion involves an increase in neural or humoral sympathetic cardiac stimulation, then propranolol should block this effect and the response to carotid hypotension after beta receptor blockade should be substantially different from that in the unblocked state in terms of the force-velocity relation and of $(\mathrm{dP} / \mathrm{dt}) / \mathrm{P}$. However, if the response to carotid occlusion involves mainly alterations in peripheral dynamics, then propanolol should have little effect on the response; the latter turned out to be the case.

To confirm the finding that carotid occlusion produces only minor changes in the inotropic state, the extent of the myocardial response to carotid occlusion was determined when the peripheral vasoconstrictor effects were minimal; the latter being prevented by the prior administration of an alpha adrenergic receptor blocking drug, phenoxybenzamine. In this situation carotid occlusion produced only a slight elevation of LV systolic pressure and a very slight increase in velocity and $(\mathrm{dP} / \mathrm{dt}) / \mathrm{P}(10 \%)$. This small increase in contractility

Carotid Sinus Control of Contractility 
was far less than the strong inotropic response elicited by norepinephrine either before or after alpha blockade (Figs. 4, 5). As an additional comparison of inotropic responses, the dose of norepinephrine was determined which would produce a similar increase in contractility to carotid occlusion after phenoxybenzamine. It was found to be only 0.01 to $0.02 \mu \mathrm{g} / \mathrm{kg}$, approximately $1 \%$ of that required to raise systolic arterial pressure by 30 to $40 \mathrm{~mm} \mathrm{Hg}$.

It has been suggested that carotid hypotension might result in hypoxia of the central nervous system, which could mask the effects of baroreceptor stimulation (9, 10). This possibility was tested in this study by observing the response to carotid occlusion in a conscious dog after recovery from carotid sinus denervation. Before denervation this animal exhibited a typical pressor response, but after section of the carotid sinus nerve, $\mathrm{BCO}$ for periods up to $1 \mathrm{~min}$ resulted in no change in $\mathrm{LV}$ pressure, velocity, $(\mathrm{dP} / \mathrm{dt}) / \mathrm{P}$ or heart rate. This indicates that the carotid sinus hypotension produced by carotid occlusion resulted in cardiovascular changes mediated by the carotid sinus reflex and not through cerebral ischemia.

We concluded from these observations that in the conscious dog carotid sinus hypotension does not augment myocardial contractility substantially. However, the possibility was considered that the reverse, i.e. carotid sinus hypertension or its physiologic analogue, electrical stimulation of the carotid sinus nerve, might be able to reduce sympathetic neural or humoral stimulation to the heart significantly and thereby to reduce myocardial contractility. Accordingly, the second part of this study was designed to test this possibility. CSNS in the conscious dog produced opposite results from carotid occlusion; a reduction in LV pressure, and an increase in velocity of shortening and peak aortic flow occurred without a significant effect on $(\mathrm{dP} / \mathrm{dt}) / \mathrm{P}$ or cardiac output. This response of the force-velocity relationship, as in the case of carotid hypotension, appeared to reflect primarily the alterations in peripheral resistance that also must have occurred. To test this hypothesis, nitroglycerin, a drug without inotropic action, was administered to reduce $\mathrm{LV}$ pressure to the same level as did CSNS. Like nerve stimulation, nitroglycerin, while decreasing pressure, also slightly increased velocity (Fig. 7) and did not have a significant effect on (dP/dt)/P (Fig. 8). Thus, it appeared in this case as well that the effects on myocardial contractility were only minor and were overshadowed by the more powerful effects on peripheral resistance. Studies in the presence of autonomic blocking agents were then conducted. After beta receptor blockade, CSNS produced reductions in pressure and increases in velocity similar to those in the unblocked state, again indicating that withdrawal of resting myocardial sympathetic tone is not an important mechanism in the response to CSNS. When the peripheral response to CSNS was attenuated by the prior administration of phenoxybenzamine, it was apparent that CSNS caused only a reduction in contractility, albeit a slight one. In this situation, LV pressure, velocity, and $(\mathrm{dP} / \mathrm{dt}) / \mathrm{P}$ decreased minimally. It could be argued that the techniques employed are not sensitive to reductions in the level of the contractile state. This does not appear to be the case since agents known to depress myocardial contractility, such as propranolol or pentobarbital, did so in the present investigation (Figs. 5,9 ) and in a previous study utilizing similar techniques (12).

In order to reconcile the findings of the present investigation with those of earlier workers who reported that CSNS or carotid sinus hypertension reflexly reduces myocardial contractility (1-8), the effects of CSNS were also studied in the anesthetized state and in the hypovolemic anesthetized state. Under these conditions, which resemble those of the acute preparations classically employed, the results were similar to those observed by others, i.e. a reflex reduction of contractility occurred (Fig. 9), presumably because the resting sympathetic tone to the heart had been augmented and could be withdrawn by sinus nerve stimulation.

The discrepancy between the responses to CSNS in the conscious and anesthetized, hypovolemic states may have clinical relevance. CSNS does not substantially decrease contractility in normal, healthy conscious dogs which appear to have a low resting level of sympathetic tone. However, in patients with cardiac disease this resting level of sympathetic tone may be much greater and the response to CSNS may involve a significant reduction in myocardial contractility as occurred in the anesthetized, hypovolemic dogs.

Additional evidence for the concept that the carotid sinus mechanism is of little importance in the control of the contractile state in the conscious dog can be derived from examination of the results of aortic blood flow. If the carotid sinus exerted a significant inotropic effect on the heart, then increases in cardiac output, stroke volume, and peak aortic flow velocity would be expected with carotid occlusion and decreases in those variables with CSNS. In point of fact, the reverse actually occurred (Fig. 2); when heart rate was allowed to vary, carotid occlusion resulted in decreases in stroke volume and peak aortic flow, while CSNS produced the opposite results (Fig. 2). In both cases cardiac output did not vary significantly, a finding in the conscious dog that we previously reported for CSNS (22) and which was noted by Fronek for carotid occlusion (29).

While this study has found that the carotid sinuses appear to exert little effect on the myocardial contrac- 
tile state in the conscious dog, it should not be interpreted to indicate that other receptors, such as those in the aortic arch, the left ventricle, and "low pressure receptors" in the atria, lungs, and great veins do not exert such effects. Indeed, studies in our laboratory (22) and by others (30) have shown that the aortic baroreceptors play a more important role than the carotid sinuses in the control of the chronotropic state of the heart, and similar consideration may apply to the inotropic state as well.

The technique of CSNS can be criticized: first, in that the carotid sinus nerve is a mixed nerve and chemoreceptor stimulation may result; and secondly, that during the maximal depressor response after autonomic blockade heart rate was slightly depressed. It is unlikely that significant chemoreceptor effects occurred since we have previously measured arterial blood gases and found no significant change in $\mathrm{P}_{2}, \mathrm{P}_{\mathrm{co}_{2}}$, and $\mathrm{pH}$ with CSNS (21). Furthermore, chemoreceptor stimulation is considered to cause no change (31) or to decrease myocardial contractility (32), and therefore could not have masked the observed response. The slight bradycardia that occurred with CSNS after alpha blockade should not significantly affect the results, and furthermore, bradycardia is thought to diminish myocardial contractility (33-35). Thus, bradycardia or chemoreceptor stimulation would tend to intensify any negative inotropic effects. Since only slight negative inotropic effects could be elicited even after the changes in peripheral resistance were minimized, it is unlikely that either of these two influences introduced serious error.

Another criticism to this technique has been the question of the adequacy of coronary blood flow. We have previously shown that coronary flow is maintained in the conscious dog during CSNS when pressure declines and that a significant coronary vasodilatation occurs (21). Feigl has found that coronary blood flow increases with carotid occlusion (36) and this was confirmed for the conscious dog in two experiments in the present study. Thus, coronary blood flow is maintained in either of the experimental situations.

The finding in this study that CSNS does not involve a substantial inotropic response mediated by the sympathetic nervous system in conscious animals is in accord with a previous work from this laboratory (11) and that of Scher and Young (37) which showed the chronotropic changes that occur with elevation of arterial pressure (37) or with CSNS (11) to be mediated primarily by an increase in vagal restraint and to a much lesser extent by a reduction in sympathetic tone. In the latter study when the animals were tested after general anesthesia, the vagal component was minimal and a profound sympathetic component appeared
(11). These findings that baroreceptor-induced cardiac slowing is primarily parasympathetically mediated have been extended to conscious man as well (38).

The previous studies demonstrating that the carotid sinus reflex is an important regulator of myocardial contractility have been conducted in anesthetized, openchest preparations (1-8). It is possible that recent surgical manipulations and the administration of a general anesthetic agent could alter the myocardial contractile state, the level of resting cardiac autonomic tone, and the central nervous system's regulation of the circulation response to the carotid sinus reflex. There is increasing evidence that the central nervous system does modulate the circulatory responses of the carotid sinus reflex (39-43), and we have previously demonstrated a profound difference in the response to CSNS before and after general anesthesia (11). We have also recently demonstrated the extent to which the myocardial contractile state is depressed by an anesthetic agent and how this can radically alter the response to an inotropic agent, such as a cardiac glycoside (12). Thus, it is probable that the carotid sinus reflex does play an important role in the regulation of myocardial contractility in anesthetized open-chest preparations. However, in the normal conscious animal the carotid sinus reflex regulates the circulation primarily through alterations in peripheral vascular resistance and produces only minor changes in the myocardial contractile state.

\section{ACKNOWLEDGMENTS}

We are grateful to D. P. McKown, D. Hendricks, and F. Werner for technical assistance.

This work was supported by U. S. Public Health Service Grants HE12373 and HE13441.

\section{REFERENCES}

1. Sarnoff, S. J., J. P. Gilmore, S. K. Brockman, J. H. Mitchell, and R. J. Linden. 1960. Regulation of ventricular contraction by the carotid sinus. Its effects on atrial and ventricular dynamics. Circ. Res. 8: 1123.

2. Polosa, C., and G. Rossi. 1961. Cardiac output and peripheral blood flow during occlusion of carotid arteries. Amer. J. Physiol. 200: 1185.

3. De Geest, H., M. N. Levy, and H. Zieske, Jr. 1964. Carotid sinus baroreceptor reflex effects upon myocardial contractility. Circ. Res. 15: 327.

4. Manning, J. W., and P. Lindgren. 1966. Sinus baroreceptor reflex and ouabain interaction on cardiac contractility. Cardiologia. 49: 345.

5. Iriuchijima, J., M. E. Soulsby, and M. F. Wilson. 1968. Participation of cardiac sympathetics in carotid occlusion pressor reflex. Amer. J. Physiol. 215: 1111.

6. Wang, H. H., C. Y. Chai, J. S. Kuo, and S. C. Wang. 1970. Participation of cardiac and peripheral sympathetics in carotid occlusion response. Amer. J. Physiol. 218: 1548 .

Carotid Sinus Control of Contractility 1007 
7. Glick, G. 1971. Importance of the carotid sinus baroreceptors in the regulation of myocardial performance. J. Clin. Invest. 50: 1116.

8. Sarnoff, S. J., and J. H. Mitchell. 1962. The control of the function of the heart. Handb. Physiol. 1 (Sec. 2): 489.

9. Salisbury, P. F., C. E. Cross, and P. A. Rieben. 1962. Regulation of ventricular and atrial contraction by carotid sinus. Circ. Res. 11: 53 .

10. Downing, S. E., and T. H. Gardner. 1968. Cephalic and carotid reflex influences on cardiac function. Amer. J. Physiol. 215: 1192.

11. Vatner, S. F., D. Franklin, and E. Braunwald. 1971. Effect of anesthesia and sleep on the circulatory response to carotid sinus nerve stimulation. Amer. J. Physiol. 220: 1249.

12. Vatner, S. F., C. B. Higgins, T. Patrick, D. Franklin, and E. Braunwald. Effects of cardiac depression and of anesthesia on the myocardial action of a cardiac glycoside. J. Clin. Invest. 50: 2585.

13. Van Citters, R. L., and D. Franklin. 1966. Telemetry of blood pressure from free-ranging animals via an intravascular gauge. J. Appl. Physiol. 21: 1633.

14. Rushmer, R. F., D. L. Franklin, and R. M. Ellis. 1956. Left ventricular dimensions recorded by sonocardiometry. Circ. Res. 4: 684 .

15. Franklin, D. L., R. L. Van Citters, and R. F. Rushmer. 1962. Left ventricular function described in physical terms. Circ. Res. 11: 702.

16. Stegall, H. F., M. B. Kardon, H. L. Stone, and V. S. Bishop. 1967. A portable, simple sonomicrometer. $J$. Appl. Physiol. 23: 289.

17. Schwartz, S. I., L. S. C. Griffith, A. Neistadt, and N. Hagfors. 1967. Chronic carotid sinus nerve stimulation in the treatment of essential hypertension. Amer. J. Surg. 114: 5 .

18. Glick, G., E. H. Sonnenblick, and E. Braunwald. 1965. Myocardial force-velocity relations studied in intact unanesthetized man. J. Clin. Invest. 44: 978.

19. Mason, D. T., E. Braunwald, E. H. Sonnenblick, J. Covell, and J. Ross, Jr. 1971. Assessment of cardiac contractility: the relation between the rate of pressure rise and ventricular pressure during isovolumic systole. Circulation. 44: 47.

20. Wolk, M. J., J. F. Keefe, O. H. L. Bing, L. J. Finkelstein, and $\mathrm{H}$. J. Levine. 1971. Estimation of $\mathrm{V}_{\max }$ in auxotonic systoles from the rate of relative increase of isovolumic pressure: $(\mathrm{dP} / \mathrm{dt}) \mathrm{KP}$. J. Clin. Invest. 50: 1276.

21. Vatner, S. F., D. Franklin, R. L. Van Citters, and E. Braunwald. 1970. Effects of carotid sinus nerve stimulation on the coronary circulation of the conscious dog. Circ. Res. 27: 11.

22. Vatner, S. F., D. Franklin, R. L. Van Citters, and E. Braunwald. 1970. Effects of carotid sinus nerve stimulation on blood-flow distribution in conscious dogs at rest and during exercise. Circ. Res. 27: 495.

23. Braunwald, E., J. Ross, Jr., and E. Sonnenblick. 1967. In Mechanisms of Contraction of the Normal and Failing Heart. Little, Brown and Co., Boston, Mass. 205.

24. Hill, A. V. 1938. The heat of shortening and the dy- namic constants of muscle. Proc. Roy. Soc. Ser. B. Biol. Sci. 126: 136

25. Abbot, B. C., and W. F. H. M. Mommaerts. 1959. A study of inotropic mechanisms in the papillary muscle preparation. J. Gen. Physiol. 42 : 533.

26. Sonnenblick, E. H. 1962. Force-velocity relations in mammalian heart muscle. Amer. J. Physiol. 202: 931.

27. Levine, H. J., and N. A. Britman. 1964. Force-velocity relations in the intact dog heart. J. Clin. Invest. 43: 1383

28. Ross, J., Jr., J. W. Covell, E. H. Sonnenblick, and E. Braunwald. 1966. Contractile state of the heart characterized by force-velocity relations in variably afterloaded and isovolumic beats. Circ. Res. 18: 149.

29. Fronek, A. 1970. Combined effect of carotid sinus hypotension and digestion on splanchnic circulation. Amer. J. Physiol. 219: 1759.

30. Glick, G., and J. W. Covell. 1968. Relative importance of the carotid and aortic baroreceptors in the reflex control of heart rate. Amer. J. Physiol. 214: 955.

31. Stern, S., and E. Rapaport. 1967. Comparison of the reflexes elicited from combined or separate stimulation of the aortic and carotid chemoreceptors on myocardial contractility, cardiac output and systemic resistance. Circ. Res. 20: 214.

32. Downing, S. E., J. P. Remensynder, and J. H. Mitchell. 1962. Cardiovascular responses to hypoxic stimulation of the carotid bodies. Circ. Res. 10: 676 .

33. Mitchell, J. H., A. G. Wallace, and N. S. Skinner, Jr. 1963. Intrinsic effects of heart rate on left ventricular performance. Amer. J. Physiol. 205: 41.

34. Boerth, R. C., J. W. Covell, P. E. Pool, and J. Ross, Jr. 1969. Increased myocardial oxygen consumption and contractile state associated with increased heart rate in dogs. Circ. Res. 24: 725.

35. Covell, J. W., J. Ross, Jr., R. Taylor, E. H. Sonnenblick, and E. Braunwald. 1967. Effects of increasing frequency of contraction on the force-velocity relation of left ventricle. Cardiovasc. Res. 1: 2.

36. Feigl, E. O. 1968. Carotid sinus reflex control of coronary blood flow. Circ. Res. 23: 223.

37. Sher, A. M., and A. C. Young. 1970. Reflex control of heart rate in the unanesthetized dog. Amer. J. Physiol. 218: 780.

38. Eckberg, D. L., M. Drabinsky, and E. Braunwald. 1971. Defective cardiac parasympathetic control in patients with heart disease. N. Engl. J. Med. 285: 877.

39. Smith, O. A., Jr., and M. A. Nathan. 1966. Inhibition of the carotid sinus reflex by stimulation of the inferior olive. Science. 154: 674 .

40. Reis, D. J., and M. Cuenod. 1965. Central neural regulation of carotid baroreceptor reflexes in the cat. Amer. J. Physiol. 209: 1267.

41. Gebber, G. L., and D. W. Snyder. 1970. Hypothalamic control of baroreceptor reflexes. Amer. J. Physiol. 218: 124

42. Klevans, L. R., and G. L. Gebber. 1970. Facilitatory forebrain influence on cardiac component of baroreceptor reflexes. Amer. J. Physiol. 219: 1235.

43. Hockman, C. H., Jr. Talesnik, and K. E. Livingston. 1969. Central nervous system modulation of baroreceptor reflexes. Amer. J. Physiol. 217: 1681. 